\title{
SUMMABILITY OF MULTILINEAR FORMS ON CLASSICAL SEQUENCE SPACES
}

\author{
T. NOGUEIRA AND P. RUEDA
}

\begin{abstract}
We present an extension of the Hardy-Littlewood inequality for multilinear forms. More precisely, let $\mathbb{K}$ be the real or complex scalar field and $m, k$ be positive integers with $m \geq k$ and $n_{1}, \ldots, n_{k}$ be positive integers such that $n_{1}+\cdots+n_{k}=m$.

(a) If $(r, p) \in(0, \infty) \times[2 m, \infty]$ then there is a constant $D_{m, r, p, k}^{\mathbb{K}} \geq 1$ (not depending on $n$ ) such that

$$
\left(\sum_{i_{1}, \ldots, i_{k}=1}^{n}\left|T\left(e_{i_{1}}^{n_{1}}, \ldots, e_{i_{k}}^{n_{k}}\right)\right|^{r}\right)^{\frac{1}{r}} \leq D_{m, r, p, k}^{\mathbb{K}} \cdot n^{\max \left\{\frac{2 k p-k p r-p r+2 r m}{2 p r}, 0\right\}}\|T\|
$$

for all $m$-linear forms $T: \ell_{p}^{n} \times \cdots \times \ell_{p}^{n} \rightarrow \mathbb{K}$ and all positive integers $n$. Moreover, the exponent $\max \left\{\frac{2 k p-k p r-p r+2 r m}{2 p r}, 0\right\}$ is optimal.

(b) If $(r, p) \in(0, \infty) \times(m, 2 m]$ then there is a constant $D_{m, r, p, k}^{\mathbb{K}} \geq 1$ (not depending on $n$ ) such that

$$
\left(\sum_{i_{1}, \ldots, i_{k}=1}^{n}\left|T\left(e_{i_{1}}^{n_{1}}, \ldots, e_{i_{k}}^{n_{k}}\right)\right|^{r}\right)^{\frac{1}{r}} \leq D_{m, r, p, k}^{\mathbb{K}} \cdot n^{\max \left\{\frac{p-r p+r m}{p r}, 0\right\}}\|T\|
$$

for all $m$-linear forms $T: \ell_{p}^{n} \times \cdots \times \ell_{p}^{n} \rightarrow \mathbb{K}$ and all positive integers $n$. Moreover, the exponent $\max \left\{\frac{p-r p+r m}{p r}, 0\right\}$ is optimal.

The case $k=m$ recovers a recent result due to G. Araujo and D. Pellegrino.
\end{abstract}

\section{INTRODUCTION}

Let $\mathbb{K}$ be $\mathbb{R}$ or $\mathbb{C}, m$ be a positive integer and $p_{1}, \ldots, p_{m} \in[1, \infty]$. For $\mathbf{p}:=\left(p_{1}, \ldots, p_{m}\right) \in[1, \infty]^{m}$, let

$$
\left|\frac{1}{\mathbf{p}}\right|:=\frac{1}{p_{1}}+\cdots+\frac{1}{p_{m}},
$$

and let us denote $X_{p}:=\ell_{p}$, when $1 \leq p<\infty$, and $X_{\infty}:=c_{0}$. The following problem has been investigated since the $30^{\prime} s$ and has important applications:

What is the best value of $\rho$ such that there is a constant $C_{\rho, \mathbf{p}}^{\mathbb{K}}$ such that

$$
\left(\sum_{j_{1}, \ldots, j_{m}=1}^{n}\left|T\left(e_{j_{1}}, \ldots, e_{j_{m}}\right)\right|^{\rho}\right)^{\frac{1}{\rho}} \leq C_{\rho, \mathbf{p}}^{\mathbb{K}}\|T\|
$$

for all continuous $m$-linear forms $T: X_{p_{1}} \times \cdots \times X_{p_{m}} \rightarrow \mathbb{K}$ and all positive integers $n$ ?

The answer is divided in some cases; for instance:

(1) $\rho=\frac{2 m}{m+1}$, when $\mathbf{p}=(\infty, \ldots, \infty) \quad$ (Bohnenblust-Hille, [ [ ] $)$;

(2) $\rho=\frac{2 m}{m+1-2\left|\frac{1}{\mathbf{p}}\right|}$, when $\left|\frac{1}{\mathbf{p}}\right| \leq \frac{1}{2} \quad$ (Hardy-Littlewood [10] and Praciano-Pereira [13]);

(3) $\rho=\frac{1}{1-\left|\frac{1}{\mathbf{p}}\right|}$, when $\frac{1}{2} \leq\left|\frac{1}{\mathbf{p}}\right|<1 \quad$ (Hardy-Littlewood [10] and Dimant-Sevilla-Peris [8]);

(4) $\rho=1$, when $\mathbf{p}=(\infty, \ldots, \infty)$ and $j_{1}=\cdots=j_{m}=j \quad$ (Aron and Globevnik [5]);

(5) $\rho=\frac{1}{1-\left|\frac{1}{\mathbf{p}}\right|}$, when $\left|\frac{1}{\mathbf{p}}\right|<1$ and $j_{1}=\cdots=j_{m}=j \quad$ (Zalduendo [14]).

Key words and phrases. multilinear forms; summability.

T. Nogueira is supported by Capes. 
These results were successfully unified in a unique inequality in [2], thanks to consider repeated indexes $j_{k}$ in the summands. Let $n_{1}, \ldots, n_{k}$ be positive integers and $n_{1}+\cdots+n_{k}=m$, and let us denote by $\left(e_{i_{1}}^{n_{1}}, \ldots, e_{i_{k}}^{n_{k}}\right)$ the $m$-tuple

$$
\left(e_{i_{1}},{ }^{n_{1}} \cdot \stackrel{\text { times }}{*}, e_{i_{1}}, \ldots, e_{i_{k}},{ }^{n_{k}} \cdot \stackrel{\text { times }}{*}, e_{i_{k}}\right) .
$$

In 2 the following result is proved:

Theorem 1 (Albuquerque et al. [2]). Let $m \geq k \geq 1, m<p \leq \infty$ and let $n_{1}, \ldots, n_{k} \geq 1$ be such that $n_{1}+\cdots+n_{k}=m$. Then, for every continuous $m$-linear form $T: X_{p} \times \cdots \times X_{p} \rightarrow \mathbb{K}$, there is a constant $M(k, m, p, \mathbb{K}) \geq 1$ such that

$$
\left(\sum_{i_{1}, \ldots, i_{k}=1}^{\infty}\left|T\left(e_{i_{1}}^{n_{1}}, \ldots, e_{i_{k}}^{n_{k}}\right)\right|^{\rho}\right)^{\frac{1}{\rho}} \leq M(k, m, p, \mathbb{K})\|T\|,
$$

with

$$
\rho=\frac{p}{p-m} \text { for } m<p \leq 2 m \text { and } M(k, m, p, \mathbb{K}) \leq C_{k, p}^{\mathbb{K}}
$$

and

$$
\rho=\frac{2 k p}{k p+p-2 m} \text { for } p \geq 2 m \text { and } M(k, m, p, \mathbb{K}) \leq D_{k, p}^{\mathbb{K}} .
$$

Moreover, in both cases, the exponent $\rho$ is optimal.

The optimality of the exponent in (1.2), implies that no constant independent of $n$ can be found for all $m$-linear forms when a smaller exponent $r$ is considered. Our objective is to show that, even for smaller exponents $r$, the value of the left hand sum increases in $n$ under control, with an explicit dependence on a power factor of $n$. We give exactly the optimal exponent for $n$. Some previous incursions to this new approach have been done in [7, Corollary 5.20]. However, it is in [3] where this subject has been first explored in its own; there, Hardy-Littlewood type inequalities have been considered and that paper has been the trigger of our work. More recently, in 9] inequalities involving homogeneous polynomials are studied and the asymptotic behavior of the constants whenever the number of variables tends to infinity is established.

This paper is a natural continuation of 2] and, in some sense it is also related to the notion of index of summability introduced by Maia, Pellegrino and Santos 11, which essentially investigates what dependence on $n$ emerges when we perturb some well known inequalities (for instance in (1.1)).

The main result of the present paper is the following:

Theorem 2. Let $m, k$ be positive integers, $m \geq k$, and let $n_{1}, \ldots, n_{k}$ be positive integers such that $n_{1}+\cdots+n_{k}=m$.

(a) If $(r, p) \in(0, \infty) \times[2 m, \infty]$ then there is a constant $D_{m, r, p, k}^{\mathbb{K}} \geq 1$ (not depending on $n$ ) such that

$$
\left(\sum_{i_{1}, \ldots, i_{k}=1}^{n}\left|T\left(e_{i_{1}}^{n_{1}}, \ldots, e_{i_{k}}^{n_{k}}\right)\right|^{r}\right)^{\frac{1}{r}} \leq D_{m, r, p, k}^{\mathbb{K}} \cdot n^{\max \left\{\frac{2 k p-k p r-p r+2 r m}{2 p r}, 0\right\}}\|T\|
$$

for all m-linear forms $T: X_{p} \times \cdots \times X_{p} \rightarrow \mathbb{K}$ and all positive integers $n$. Moreover, the exponent $\max \left\{\frac{2 k p-k p r-p r+2 r m}{2 p r}, 0\right\}$ is optimal.

(b) If $(r, p) \in(0, \infty) \times(m, 2 m]$ then there is a constant $D_{m, r, p, k}^{\mathbb{K}} \geq 1$ (not depending on $n$ ) such that

$$
\left(\sum_{i_{1}, \ldots, i_{k}=1}^{n}\left|T\left(e_{i_{1}}^{n_{1}}, \ldots, e_{i_{k}}^{n_{k}}\right)\right|^{r}\right)^{\frac{1}{r}} \leq D_{m, r, p, k}^{\mathbb{K}} \cdot n^{\max \left\{\frac{p-r p+r m}{p r}, 0\right\}}\|T\|
$$

for all m-linear forms $T: X_{p} \times \cdots \times X_{p} \rightarrow \mathbb{K}$ and all positive integers $n$. Moreover, the exponent $\max \left\{\frac{p-r p+r m}{p r}, 0\right\}$ is optimal. 


\section{THE PROOF}

Let $E_{1}, \ldots, E_{m}$ be Banach spaces. The product $\hat{\otimes}_{j \in\{1, \ldots, m\}}^{\pi} E_{j}=E_{1} \hat{\otimes}^{\pi} \cdots \hat{\otimes}^{\pi} E_{m}$ denotes the $m$ fold completed projective tensor product of $E_{1}, \ldots, E_{m}$. The tensor $x_{1} \otimes \cdots \otimes x_{m}$ will be denoted by $\otimes_{j \in\{1, \ldots, m\}} x_{j}$, and $\otimes_{m} x$ shall denote the tensor $x \otimes \cdots \otimes x$.

Define $\frac{1}{r_{j}}=\frac{n_{j}}{p}$ and note that $\frac{1}{r_{j}}<1$ for all $j=1, \ldots, k$ (because $p>m$ ). Let $D_{r_{j}} \subset X_{p} \hat{\otimes}^{\pi} \cdots \hat{\otimes}^{\pi} X_{p}$ $\left(n_{j}\right.$ times) be the vector space generated by the tensors $\otimes_{n_{j}} e_{i}$ and consider the isometric isomorphism (see [4] and 2]) $u_{j}: X_{r_{j}} \rightarrow \overline{D_{r_{j}}}$ defined by

$$
u_{j}\left(\sum_{i=1}^{\infty} a_{i} e_{i}\right)=\sum_{i=1}^{\infty} a_{i} \otimes_{n_{j}} e_{i} .
$$

For any continuous $m$-linear form $T: X_{p} \times \cdots \times X_{p} \rightarrow \mathbb{K}$, consider its $k$-linearization $\widehat{T}$ : $\otimes_{n_{1}}^{\pi} X_{p} \times \cdots \times \otimes_{n_{k}}^{\pi} X_{p}$, that is, $\widehat{T}$ is the unique $k$-linear form such that $\widehat{T}\left(x_{1}^{1} \otimes \cdots \otimes x_{n_{1}}^{1}, \ldots, x_{1}^{k} \otimes \cdots \otimes x_{n_{k}}^{k}\right)=$ $T\left(x_{1}^{1}, \ldots, x_{n_{1}}^{1}, \ldots, x_{1}^{k}, \ldots, x_{n_{k}}^{k}\right)$ for all $x_{j}^{i} \in X_{p}, 1 \leq j \leq n_{i}, 1 \leq i \leq k$ (for further details we refer to [2]), and let $S: X_{r_{1}} \times \cdots \times X_{r_{k}} \rightarrow \mathbb{K}$ be given by

$$
S\left(w_{1}, \ldots, w_{k}\right):=\widehat{T}\left(u_{1}\left(w_{1}\right), \ldots, u_{k}\left(w_{k}\right)\right) .
$$

Then

$$
\begin{aligned}
\left(\sum_{i_{1}, \ldots, i_{k}=1}^{n}\left|T\left(e_{i_{1}}^{n_{1}}, \ldots, e_{i_{k}}^{n_{k}}\right)\right|^{r}\right)^{\frac{1}{r}} & =\left(\sum_{i_{1}, \ldots, i_{k}=1}^{n}\left|\widehat{T}\left(u_{1}\left(e_{i_{1}}\right), \ldots, u_{k}\left(e_{i_{k}}\right)\right)\right|^{r}\right)^{\frac{1}{r}} \\
& =\left(\sum_{i_{1}, \ldots, i_{k}=1}^{n}\left|S\left(e_{i_{1}}, \ldots, e_{i_{k}}\right)\right|^{r}\right)^{\frac{1}{r}} .
\end{aligned}
$$

Proof of (a). Let us first suppose that $(r, p) \in\left(0, \frac{2 k p}{k p+p-2 m}\right] \times[2 m, \infty]$. Using the Hölder inequality and Theorem 1 we have

$$
\begin{aligned}
& \left(\sum_{i_{1}, \ldots, i_{k}=1}^{n}\left|T\left(e_{i_{1}}^{n_{1}}, \ldots, e_{i_{k}}^{n_{k}}\right)\right|^{r}\right)^{\frac{1}{r}} \\
& \leq\left(\sum_{i_{1}, \ldots, i_{k}=1}^{n}\left|T\left(e_{i_{1}}^{n_{1}}, \ldots, e_{i_{k}}^{n_{k}}\right)\right|^{\frac{2 k p}{k_{p+p-2 m}}}\right)^{\frac{k p+p-2 m}{2 k p}} \cdot\left(\sum_{i_{1}, \ldots, i_{k}=1}^{n}|1|^{\frac{2 k p-r k p-r p+2 m r}{2 k p}}\right)^{\frac{2 k p-r k p-r p+2 m r}{2 k p r}} \\
& =\left(\sum_{i_{1}, \ldots, i_{k}=1}^{n}\left|T\left(e_{i_{1}}^{n_{1}}, \ldots, e_{i_{k}}^{n_{k}}\right)\right|^{\frac{2 k p}{k p+p-2 m}}\right)^{\frac{k p+p-2 m}{2 k p}} \cdot\left(n^{k}\right)^{\frac{2 k p-r k p-r p+2 m r}{2 k p r}} \\
& \leq M(k, m, p, \mathbb{K})\|T\| \cdot\left(n^{k}\right)^{\frac{2 k p-r k p-r p+2 m r}{2 k p r}} \\
& =M(k, m, p, \mathbb{K})\|T\| \cdot n^{\frac{2 k p-r k p-r p+2 m r}{2 p r}} .
\end{aligned}
$$

On the other hand, if $(r, p) \in\left[\frac{2 k p}{k p+p-2 m}, \infty\right] \times[2 m, \infty]$, we have

$$
\begin{aligned}
& \left(\sum_{i_{1}, \ldots, i_{k}=1}^{n}\left|T\left(e_{i_{1}}^{n_{1}}, \ldots, e_{i_{k}}^{n_{k}}\right)\right|^{r}\right)^{\frac{1}{r}} \\
& \leq\left(\sum_{i_{1}, \ldots, i_{k}=1}^{\infty}\left|T\left(e_{i_{1}}^{n_{1}}, \ldots, e_{i_{k}}^{n_{k}}\right)\right|^{\frac{2 k p}{k p+p-2 m}}\right)^{\frac{k p+p-2 m}{2 k p}} \\
& \leq M(k, m, p, \mathbb{K})\|T\| \\
& =M(k, m, p, \mathbb{K})\|T\| \cdot n^{\max \left\{\frac{2 k p-r k p-r p+2 m r}{2 p r}, 0\right\}}
\end{aligned}
$$


and, of course, in this case the exponent $\max \left\{\frac{2 k p-r k p-r p+2 m r}{2 p r}, 0\right\}$ is optimal.

It remains to prove the optimality of the exponent in the case $(r, p) \in\left(0, \frac{2 k p}{k p+p-2 m}\right] \times[2 m, \infty]$. We shall use a technique used in the main result of [2]. Suppose that $\lambda \geq 0$ is the smallest exponent satisfying

$$
\left(\sum_{i_{1}, \ldots, i_{k}=1}^{n}\left|T\left(e_{i_{1}}^{n_{1}}, \ldots, e_{i_{k}}^{n_{k}}\right)\right|^{r}\right)^{\frac{1}{r}} \leq D_{m, r, p, k}^{\mathbb{K}} \cdot n^{\lambda}\|T\|
$$

for all continuous $m$-linear forms $T: X_{p} \times \cdots \times X_{p} \rightarrow \mathbb{K}$. Let us show that $\lambda=\max \left\{\frac{2 m r+2 k p-k p r-p r}{2 p r}, 0\right\}$. Let $A: X_{r_{1}} \times \cdots \times X_{r_{k}} \rightarrow \mathbb{K}$ be a continuous $k$-linear form. For each $i=1, \ldots, k$ we know that $\bar{D}_{r_{i}}$ is complemented into $\hat{\otimes}_{j \in\{1, \ldots, m\}}^{\pi} X_{p}$, and consider the canonical projection $d_{r_{i}}: \hat{\otimes}_{j \in\{1, \ldots, m\}}^{\pi} X_{p} \rightarrow \bar{D}_{r_{i}}$ (see 4 for details). Defining the $m$-linear form $T_{A}: X_{p} \times \cdots \times X_{p} \rightarrow \mathbb{K}$ by

$$
T_{A}\left(x_{1}^{(1)}, \ldots, x_{n_{1}}^{(1)}, \ldots, x_{1}^{(k)}, \ldots, x_{n_{k}}^{(k)}\right):=A\left(u_{r_{1}}^{-1} \circ d_{r_{1}}\left(x_{1}^{(1)} \otimes \cdots \otimes x_{n_{1}}^{(1)}\right), \ldots, u_{r_{k}}^{-1} \circ d_{r_{k}}\left(x_{1}^{(k)} \otimes \cdots \otimes x_{n_{k}}^{(k)}\right)\right),
$$
we have

$$
\begin{aligned}
T_{A}\left(e_{i_{1}}^{n_{1}}, \ldots, e_{i_{k}}^{n_{k}}\right) & =A\left(u_{r_{1}}^{-1} \circ d_{r_{1}}\left(\otimes_{n_{1}} e_{i_{1}}\right), \ldots, u_{r_{k}}^{-1} \circ d_{r_{k}}\left(\otimes_{n_{k}} e_{i_{k}}\right)\right) \\
& =A\left(u_{r_{1}}^{-1}\left(\otimes_{n_{1}} e_{i_{1}}\right), \ldots, u_{r_{k}}^{-1}\left(\otimes_{n_{k}} e_{i_{k}}\right)\right)=A\left(e_{i_{1}}, \ldots, e_{i_{k}}\right) .
\end{aligned}
$$

By (2.3) and (2.2) applied to $T_{A}$, and using that $\left\|T_{A}\right\| \leq\|A\|$, we obtain

$$
\left(\sum_{i_{1}, \ldots, i_{k}=1}^{n}\left|A\left(e_{i_{1}}, \ldots, e_{i_{k}}\right)\right|^{r}\right)^{\frac{1}{r}} \leq D_{m, r, p, k}^{\mathbb{K}} \cdot n^{\lambda}\|A\|
$$

Since $A$ is $k$-linear, and

$$
\frac{1}{r_{j}}=\frac{n_{j}}{p} \leq \frac{m}{2 m}=\frac{1}{2}
$$

from the Kahane-Salem-Zygmund inequality (see [1, Lemma 6.1] for details), there is a constant $C_{k}>0$ such that

Making $n \rightarrow \infty$, we have

$$
n^{\frac{k}{r}} \leq C_{k} M_{k, r, r_{1}, \ldots, r_{k}}^{\mathbb{K}} n^{\lambda} n^{\frac{k+1}{2}-\left(\frac{1}{r_{1}}+\cdots+\frac{1}{r_{k}}\right)}
$$

$$
\lambda \geq \frac{1}{r_{1}}+\cdots+\frac{1}{r_{k}}+\frac{2 k-k r-r}{2 r} .
$$

Since $\frac{1}{r_{1}}+\cdots+\frac{1}{r_{k}}=\frac{m}{p}$, we have

$$
\lambda \geq \frac{m}{p}+\frac{2 k-k r-r}{2 r}=\max \left\{\frac{2 m r+2 k p-k p r-p r}{2 p r}, 0\right\} .
$$

Proof of (b). Since

$$
\left(\sum_{i_{1}, \ldots, i_{k}=1}^{n}\left|T\left(e_{i_{1}}^{n_{1}}, \ldots, e_{i_{k}}^{n_{k}}\right)\right|^{r}\right)^{\frac{1}{r}} \leq\left(\sum_{j_{1}, \ldots, j_{m}=1}^{n}\left|T\left(e_{j_{1}}, \ldots, e_{j_{m}}\right)\right|^{r}\right)^{\frac{1}{r}},
$$

by [3, Theorem 1.1(b)] we have

$$
\left(\sum_{i_{1}, \ldots, i_{k}=1}^{n}\left|T\left(e_{i_{1}}^{n_{1}}, \ldots, e_{i_{k}}^{n_{k}}\right)\right|^{r}\right)^{\frac{1}{r}} \leq D_{m, r, p, k}^{\mathbb{K}} \cdot n^{\max \left\{\frac{p-r p+r m}{p r}, 0\right\}}\|T\| .
$$

Let us prove the optimality of the exponent. If

$$
\frac{m r+p-p r}{p r} \leq 0
$$

the optimality of the exponent $\max \{(m r+p-p r) / p r, 0\}$ is immediate.

Suppose that the inequality holds for a certain exponent $s \geq 0$; thus

$$
\left(\sum_{i_{1}, \ldots, i_{k}=1}^{n}\left|T\left(e_{i_{1}}^{n_{1}}, \ldots, e_{i_{k}}^{n_{k}}\right)\right|^{r}\right)^{\frac{1}{r}} \leq D_{m, r, p, k}^{\mathbb{K}} \cdot n^{s}\|T\|
$$


As in the previous case, for each continuous $k$-linear form $A: X_{r_{1}} \times \cdots \times X_{r_{k}} \rightarrow \mathbb{K}$, with $r_{j}=\frac{p}{n_{j}}$, and for all $j=1, \ldots, k$, there is a continuous $m$-linear form $T_{A}: X_{p} \times \cdots \times X_{p} \rightarrow \mathbb{K}$ such that

$$
T_{A}\left(e_{i_{1}}^{n_{1}}, \ldots, e_{i_{k}}^{n_{k}}\right)=A\left(e_{i_{1}}, \ldots, e_{i_{k}}\right)
$$

and $\left\|T_{A}\right\| \leq\|A\|$. By (2.5) and (2.4) applied to $T_{A}$ we obtain

$$
\left(\sum_{i_{1}, \ldots, i_{k}=1}^{n}\left|A\left(e_{i_{1}}, \ldots, e_{i_{k}}\right)\right|^{r}\right)^{\frac{1}{r}} \leq D_{m, r, p, k}^{\mathbb{K}} \cdot n^{s}\left\|T_{A}\right\| \leq D_{m, r, p, k}^{\mathbb{K}} \cdot n^{s}\|A\| .
$$

Define the $k$-linear form $S: X_{r_{1}} \times \cdots \times X_{r_{k}} \rightarrow \mathbb{K}$ by

$$
S\left(x^{(1)}, \ldots, x^{(k)}\right)=\sum_{j=1}^{n} x_{j}^{(1)} \cdots x_{j}^{(k)},
$$

and notice that by the Hölder inequality we have

$$
\|S\| \leq n^{1-\left(\frac{1}{r_{1}}+\cdots+\frac{1}{r_{k}}\right)}=n^{1-\frac{m}{p}} .
$$

Therefore, plugging $S$ into (2.6) we get

$$
n^{\frac{1}{r}} \leq D_{m, r, p, k}^{\mathbb{K}} n^{s} n^{1-\frac{m}{p}}
$$

and we easily conclude that

$$
s \geq \frac{p-r p+r m}{p r}
$$

\section{REFERENCES}

[1] N. Albuquerque, F. Bayart, D. Pellegrino and J. Seoane-Sepúlveda, Sharp generalizations of the multilinear Bohnenblust-Hille inequality, J. Funct. Anal. 266 (2014), 3726-3740.

[2] N. Albuquerque, G. Araújo, D. Núñez-Alarcón, D. Pellegrino, and P. Rueda, Bohnenblust-Hille and Hardy-Littlewood inequalities by blocks, arXiv:1409.6769v6 [math.FA].

[3] G. Araújo, D. Pellegrino, Optimal Hardy-Littlewood type inequalities for $m$-linear forms on $\ell_{p}$ spaces with $1 \leq p \leq m$, Archiv der Math. 105 (2015), 285-295.

[4] A. Arias, J. D. Farmer, On the structure of tensor products of $\ell_{p}$-spaces, Pacific J. Math. 175 (1996), 13-37.

[5] R.M. Aron, J. Globevnik, Analytic functions on $c_{0}$. Congress on Functional Analysis (Madrid, 1988). Rev. Mat. Univ. Complut. Madrid 2 (1989), suppl., 27-33.

[6] H.F. Bohnenblust and E. Hille, On the absolute convergence of Dirichlet series, Ann. of Math. (2) 32 (1931), 600-622.

[7] G. Botelho, C. Michels and D. Pellegrino, Complex interpolation and summability properties of multilinear operators, Rev. Mat. Complut. 23 (2010), 139-161.

[8] V. Dimant and P. Sevilla-Peris, Summation of coefficients of polynomials on $\ell_{p}$ spaces, arXiv:1309.6063 1 [math.FA] 24 Sep 2013.

[9] D. Galicer, M. Mansila, S. Muro, The sup-norm vs. the norm of the coefficients: equivalence constants for homogeneous polynomials. Preprint. arXiv:1602.01735v1 [math.FA] 4 Feb 2016.

[10] G. Hardy, J. E. Littlewood, Bilinear forms bounded in space [p, $q]$, Quart. J. Math. 5 (1934), 241-254.

[11] M. Maia, D. Pellegrino and J. Santos, An index of summability for pairs of Banach spaces, arXiv:1602.03363.

[12] D. Pérez-García and I. Villanueva. Multiple summing operators on C(K) spaces. Ark. Mat., 42 (2004), $153-171$.

[13] T. Praciano-Pereira, On bounded multilinear forms on a class of $\ell_{p}$ spaces, J. Math. Anal. Appl. 81 (1981), 561-568.

[14] I. Zalduendo, An estimate for multilinear forms on $\ell_{p}$, Proc. Roy. Irish Acad. Sect. A 93 (1993), no. 1, $137-142$.

(T. Nogueira) Departamento de Matemática, Universidade Federal da Paraíba, $\quad$ 58.051-900 - João Pessoa, BRAZIL.

E-mail address: tonykleverson@gmail.com

(P. Rueda) Departamento de AnÁlisis Matemático, Universidad de Valencia, 46100 Burjassot, Valencia. E-mail address: pilar.rueda@uv.es 